\title{
A Flexible Multistage Demand System Based on Indirect Separability
}

\author{
GianCarlo Moschini
}

Working Paper 00-WP 265

December 2000

\author{
Centerfor Agricultural and Rural Development \\ lowa State University \\ Ames, lowa 50011-1070 \\ www.card.iastate.edu
}

GianCarlo Moschini is professor of economics and Pioneer Hi-Bred International Chair in Science and Technology Policy, Department of Economics and Center for Agricultural and Rural Development, Iowa State University.

This publication is available online on the CARD website: www.card.iastate.edu.

For questions or comments about the contents of this paper, please contact, GianCarlo Moschini, Iowa State University, 583 Heady Hall, Ames, Iowa, 50011-1070; Ph: 515-294-5761; Fax: 515-294-6336; email: moschini@iastate.edu.

Iowa State University does not discriminate on the basis of race, color, age, religion, national origin, sexual orientation, sex, marital status, disability, or status as a U.S. Vietnam Era Veteran. Any Persons having inquiries concerning this may contact the Director of Affirmative Action, 318 Beardshear Hall, 515-294-7612. 


\begin{abstract}
The notion of indirect separability is exploited to derive a new multistage demand system. The model allows a consistent parameterization of demand relations at various budgeting stages and it fulfills the requirement of flexibility while satisfying separability globally. Two propositions are derived to characterize flexible and separable functional forms, which lead to the specification of a flexible and separable translog (FAST) demand system. The model is particularly attractive for modeling large complete demand systems, and is illustrated with an application to Canadian food demand.
\end{abstract}

JEL codes: $\quad$ D11, D12, C51. 


\section{A FLEXIBLE MULTISTATE DEMAND SYSTEM BASED ON INDIRECT SEPARABILITY}

\section{Introduction}

To be useful for most policy analysis applications, demand systems need to be specified in terms of disaggregated commodities based on relevant conditioning variables, i.e., usually one needs to specify a large complete demand system. If, at the same time, one wants to use parametric specifications that are not too constraining, such as standard "flexible functional forms" (FFF), then the data requirement may be prohibitive. A workable solution of this problem entails imposing restrictions on the problem solved by consumers, typically by assuming a separable structure for consumer preferences (Blackorby, Primont and Russell 1978). ${ }^{1}$ In particular, certain separability conditions allow the consumer's expenditure allocation problem to satisfy two-stage (multistage) budgeting rules. As shown by Gorman (1959), a simplified twostage budgeting is possible under two alternative conditions: homothetic weak separability of the direct utility function, or strong separability (block additivity) of the direct utility function with group subutility functions, the dual of which have the so-called generalized Gorman polar form (GGPF). Such "perfect price aggregation" conditions underlie a number of multistage complete demand systems. Models relying on strong separability cum GGPF include Brown and Heien (1972), Blackorby, Boyce and Russell (1978), Anderson (1979), and Yen and Roe (1989). Homothetic weak separability was used by Jorgenson, Slesnick and Stoker (1997). ${ }^{2}$

Because the conditions for perfect price aggregation are often deemed too restrictive in empirical applications, attempts have been made to model demand based on the hypothesis of direct weak separability only. Although direct weak separability (DWS), per se, is neither necessary nor sufficient for standard two-stage budgeting, it does provide the necessary and sufficient conditions for the existence of conditional (second-stage) demand functions defined only on group prices and group expenditure allocations (Pollak 1971). Because such conditional demand functions typically depend on a small set of variables (data on which is easily found for 
most applications), some empirical studies have pursued the estimation of second-stage demand functions in isolation (say, demand for food items as function of food prices and expenditure allocated to food). Examples include Barr and Cuthbertson (1994), Gao, Wailes and Cramer (1997), Kinnucan, Xiao, Hsia and Jackson (1997), and Spencer (1997). But such a widespread approach is highly questionable because the conditional demand parameters thus estimated are rarely of interest for policy analysis (Hanemann and Morey, 1992). In certain instances a conditional analysis can provide useful information (Browning and Meghir 1991). But in general the economic question at hand requires one to recover unconditional demands and, under DWS, that necessitates estimation of both first-stage and second-stage expenditure allocation functions.

If one insists on not weakening the assumption of DWS, then estimation of a complete demand system requires a consistent parametric specification for the two budgeting stages. But, whereas one can easily derive second-stage demand functions by Roy's identity from a specification of the (separable) group indirect subutility function, derivation of first-stage expenditure allocation functions requires an explicit solution of the conditional utility maximization problem (Blackorby, Primont and Russell 1978, chapter 5). It follows that an internally consistent parametric specification of the two budgeting stages is difficult when relying only on DWS (especially if one wants to satisfy a notion of flexibility). If one is willing to accept an approximate solution to the first-stage income allocation under direct weak separability, a useful approach is outlined by Gorman (1995). But implementations of such an approximation are somewhat unsatisfactory. ${ }^{3}$

This article explores an alternative route to specifying a two-stage flexible demand system by assuming that it is the indirect utility function (rather than the direct utility function) that is weakly separable. This study makes two contributions. First, it shows how indirect weak separability (IWS) can be used to specify a complete demand system that is amenable to a recursive structure typically associated with multistage budgeting. In particular, IWS still permits a meaningful definition of conditional (second-stage) demand functions. Of course, first-stage income allocations functions here cannot be specified in terms of only one price index per group (the same consideration holds for DWS), but will instead depend on all prices and total expenditure. But, unlike DWS, in this setting it is straightforward to specify simultaneously first- 
stage and second-stage demand functions, because under IWS both can be obtained by Roy's identity from a well-specified (and separable) indirect utility function. Furthermore, this specification strategy ensures that the parametric structure of the resulting system of unconditional demand functions is internally consistent. It should also be emphasized that the recursive demand system permitted by IWS does not require the restrictive assumptions of perfect price aggregation. Specifically, it does not require strong separability (additivity with respect to the subutility functions) nor does it require homotheticity of the separable subutility functions. In this sense it is a genuine generalization of existing multistage demand systems.

The second main contribution of this article concerns the parametric specification of the separable demand system. It is known that maintaining or testing separability with commonly used FFF entails difficulties. Blackorby, Primont and Russell (1977) showed that separability conditions on FFF introduce unwanted restrictions that typically destroy the flexibility of the function. The standard way to deal with this issue has been to require separability to hold only at a point (Jorgenson and Lau 1975; Denny and Fuss 1977). ${ }^{4}$ The validity of this procedure has originated considerable debate, especially when the focus is on testing the separability assumption (Aizcorbe 1992). This article proposes an appealing alternative by deriving a general procedure to specify flexible and separable functions. This procedure, applied to an indirectly separable utility function, leads to a demand model that satisfies the standard definitions of flexibility and satisfies the postulated separable structure globally. The "flexible and separable translog" (FAST) demand system thus derived is illustrated with an application to a complete demand system for Canada that emphasizes food consumption.

\section{Why Indirect Separability?}

The attractive features of IWS for the purpose of specifying a complete demand system are best illustrated by comparison with the more common assumption of DWS. To recall briefly these separability notions, let $q \equiv\left[q_{1}, q_{2}, \ldots, q_{n}\right]$ denote the vector of goods demanded by the consumer and $p \equiv\left[p_{1}, p_{2}, \ldots, p_{n}\right]$ the corresponding vector of all nominal prices. Let $U(q)$ represent the direct utility function, which is assumed continuous, non-decreasing and strictly quasiconcave. If $I=\{1,2, \ldots, n\}$ denotes the set of indices of the $n$ goods, order these goods in $N<n$ separable 
groups defined by the mutually exclusive and exhaustive partition $\hat{I}=\left\{I^{I}, I^{2}, \ldots, I^{\mathrm{N}}\right\}$ of the set I. Then (symmetric) DWS holds if $U(q)$ can be written as:

$$
U(q)=U^{0}\left(U^{1}\left(q^{1}\right), U^{2}\left(q^{2}\right), \ldots, U^{N}\left(q^{N}\right)\right)
$$

This structure on the utility function is sufficient to guarantee the existence of conditional demand functions $\tilde{c}_{i}\left(p^{r} / y_{r}\right), \forall i \in I^{r}(r=1, \ldots, N)$, where $p^{r}$ is the vector of prices in the partition $I^{r}$ (such that $p=\left[p^{1}, p^{2}, \ldots, p^{N}\right]$ ), and $y_{r}$ is the optimal allocation of expenditure to the goods in the $r^{\text {th }}$ group. These conditional demand functions can be derived by Roy's identity from the indirect subutility function that is dual to $U\left(q^{r}\right)$, say $\tilde{V}^{r}\left(p^{r} / y_{r}\right)$. Because they only require data on the particular separable group, the second-stage demand functions $\tilde{c}_{i}\left(p^{r} / y_{r}\right)$ are often estimated in applied work.

It is clear that such conditional demand functions cannot provide the parameters (i.e., elasticities) that are typically of interest for policy questions. This is because the optimal allocation of expenditure to the goods in any one partition depends on all prices and total expenditure. That is, the optimal solution to the first-stage expenditure allocation problem has the functional representation $\tilde{y}_{r}(p / y), r=1,2, \ldots, N$, where $y$ is total expenditures. ${ }^{5}$ Hence, the consumer's unconditional demand functions $q_{i}(p / y)$ satisfy $q_{i}(p / y) \equiv \tilde{c}_{i}\left(p^{r} / \tilde{y}(p / y)\right)$. To say anything meaningful about a consumer's response to change in any one price, say, one needs to know how the optimal allocations $\tilde{y}_{r}(p / y)$ are affected by this change, i.e., one needs to estimate the first-stage expenditure allocation functions as well. But if one adopts standard FFF for the groups' indirect utility functions $\tilde{V}^{r}\left(p^{r} / y_{r}\right)$ (the almost ideal or translog systems, for example), then to obtain a consistent specification for the first-stage expenditure allocation functions one needs an explicit solution of the conditional utility maximization problem (Blackorby, Primont and Russell 1978, chapter 5). For nontrivial specifications of the function $U^{0}($.$) , such an explicit (and exact) solution may not be possible.$

Now consider IWS. Let $V(p / y)$ represent the indirect utility function, where $p$ and $y$ are defined as before. Under standard regularity conditions, the indirect utility function is continuous, nonincreasing and quasiconvex in $p / y$, and homogeneous of degree zero in $(p, y)$ (a property that 
is explicitly maintained here). Then preferences are said to be (symmetrically) indirectly weakly separable in the partition $\hat{I}$ if $V(p / y)$ can be written as

$$
V(p / y)=V^{0}\left(V^{1}\left(p^{1} / y\right), V^{2}\left(p^{2} / y\right), \ldots, V^{N}\left(p^{N / y)}\right),\right.
$$

where $p^{r}(r=1, \ldots, N)$ are the group price vectors defined earlier, and $V^{r}\left(p^{r} / y\right)$ are indices that depend only on group prices and total expenditures. $V^{O}($.$) is assumed to have (with respect to the$ price indices $V^{r}$ ) the typical properties of an indirect utility function: continuity, negative monotonicity, and quasiconvexity. The functions $V^{r}($.$) , instead, are assumed to be continuous,$ nondecreasing and quasiconcave in their arguments, such that $V(p / y)$ in (1) retains the typical properties of an indirect utility function with respect to individual normalized prices $p_{i} / y$ (Blackorby, Primont and Russell 1978, p. 68). ${ }^{6}$

Indirect separability allows a potentially useful recursive characterization of the consumer's budgeting problem, what Blackorby, Primont and Russell (1978) call "weak decentralizability." Specifically, if the indirect utility function has the structure in (1), by Roy's identity the (unconditional) Marshallian demands are given by:

$$
q_{i}(p / y)=-\frac{\frac{\partial V^{0}(.)}{\partial V^{r}} \frac{\partial V^{r}\left(p^{r} / y\right)}{\partial p_{i}}}{\sum_{s=1}^{N} \frac{\partial V^{0}(.)}{\partial V^{s}} \frac{\partial V^{s}\left(p^{s} / y\right)}{\partial y}} \quad i \in I^{r}
$$

Hence, under IWS the expenditure allocation to goods in any one partition, say $y_{r}(p / y)$, must satisfy:

$$
y_{r}(p / y) \equiv \sum_{i \in I^{r}} p_{i} q_{i}(p / y)=-\frac{\frac{\partial V^{0}(.)}{\partial V^{r}} \sum_{i \in I^{r}} \frac{\partial V^{r}\left(p^{r} / y\right)}{\partial p_{i}} p_{i}}{\sum_{s=1}^{N} \frac{\partial V^{0}(.)}{\partial V^{s}} \frac{\partial V^{s}\left(p^{s} / y\right)}{\partial y}} \quad r=1,2, \ldots, N
$$


If we now define:

$$
c_{i}\left(p^{r / y}\right) \equiv-\frac{\frac{\partial V^{r}\left(p^{r} / y\right)}{\partial p_{i}}}{\frac{\partial V^{r}\left(p^{r} / y\right)}{\partial y}} \quad i \in I^{r}
$$

then, from equations (2)-(4), the Marshallian unconditional demand functions can be expressed in terms of the first-stage expenditure allocation functions $y_{r}(p / y)$, and of the second-stage (conditional) demand functions $c_{i}\left(p^{r} / y\right)$, that is:

$$
q_{i}(p / y)=\frac{y_{r}(p / y)}{y} c_{i}\left(p^{r / y}\right), \quad \quad i \in I^{r} .
$$

Equation (5) clarifies in what sense $c_{i}\left(p^{r} / y\right)$ are "conditional" demand functions: given an expenditure allocation $y_{r}$ to the group, optimal within-group allocation is possible given knowledge of only group prices $p^{r}$ and total expenditure $y$. Of course, these somewhat unusual conditional demand functions differ from the conditional demands $\tilde{c}_{i}\left(p^{r} / y_{r}\right)$ obtained under DWS. But it is important to emphasize that the functions $c_{i}\left(p^{r} / y\right)$ do have all the properties of standard Marshallian demand functions, such as symmetry and negative semidefiniteness of compensated effects (unlike the allocation functions considered by Blackorby, Primont and Russell 1978, p. 191). These attributes are apparent from (4), which shows that the functions $c_{i}\left(p^{r} / y\right)$ can be viewed as derived from a standard indirect utility function via Roy's identity (this interpretation is possible because the negative [or the reciprocal] of $V^{r}\left(p^{r} / y\right)$ behaves like an indirect utility function, as discussed earlier).

Because estimating the consumer's unconditional response is the main motive of interest here, the IWS conditional demand functions $c_{i}\left(p^{r} / y\right)$, by themselves, are not more useful than the DWS conditional demand functions $\tilde{c}_{i}\left(p^{r} / y_{r}\right)$; in both cases one needs to estimate the first-stage group allocation functions as well to recover unconditional demands. But as shown in the foregoing, a consistent specification of first- and second-stage functions is straightforward under IWS, as both can be obtained by Roy's identity given a (separable) functional form for the indirect utility 
function. This convenient attribute, which sets IWS apart from DWS, will be illustrated further with the specific translog specification to be used in the empirical application.

Given that IWS was motivated earlier as contrasted with DWS, some further comments may be useful. From the point of view of estimating a complete demand system, the main implication of either separability assumption is that each entails restrictions on the substitution possibilities between goods belonging to different partitions. As Blackorby, Primont and Russell (1998, p. 66) put it, " ... direct separability of sector $r$ means that marginal rates of substitution -- i.e., sector- $r$ shadow price ratios -- are independent of consumption quantities outside the $r^{\text {th }}$ sector; indirect separability of sector $r$ means that optimal quantity ratios in this sector are independent of prices outside the sector." Alternatively, let $\sigma_{i k}$ denote the Allen-Uzawa elasticity of substitution (Uzawa 1962) and let $\varepsilon_{i} \equiv \partial \log q_{i} / \partial \log y$ denote the (unconditional) expenditure elasticity. Then, for any two goods in the partition $I^{r}$ and any two goods in another partition $I^{S}$, under DWS one obtains (Moschini, Moro and Green 1994):

$$
\frac{\sigma_{i k}}{\sigma_{j m}}=\frac{\varepsilon_{i} \varepsilon_{k}}{\varepsilon_{j} \varepsilon_{m}} \quad \forall(i, j) \in I^{r} \quad \text { and } \quad \forall(k, m) \in I^{s}
$$

On the other hand, under IWS it is easy to show that:

$$
\sigma_{i k}-\sigma_{j m}=\varepsilon_{i}+\varepsilon_{k}-\varepsilon_{j}-\varepsilon_{m} \quad \forall(i, j) \in I^{r} \quad \text { and } \quad \forall(k, m) \in I^{s}
$$

Hence, in both instances of separability, all the cross-substitution terms between goods in two different groups can be identified based on knowledge of only one of them (given expenditure elasticities). ${ }^{7}$

\section{Flexible Functional Forms for Separable Functions}

To apply the IWS framework to the task of specifying an internally consistent set of firstand second-stage demand functions we need to specify a separable indirect utility function. Furthermore, following standard requirements, we want this function to be "flexible," subject to the restrictions implied by IWS just discussed. Moschini (1992), Driscoll and McGuirk (1992), 
and Diewert and Wales (1995) show how it is possible to construct functional forms that satisfy separability globally in a production context. In our setting, this procedure entails choosing functional forms for $V^{0}$ and all the $V^{r}$ to reflect the structure of the postulated utility function. To this end, some general results are presented that offer a systematic procedure for constructing flexible and separable functions. To begin, let us recall the following:

Definition: Flexibility (Diewert, 1974). A function $F(x)$, where $x$ is an n-vector, is a flexible functional form $(F F F)$ for a function $V(x)$ if $F(x)$ can provide a second-order approximation to $V(x)$ at a point $\bar{x}$. In other words, $F(x)$ must be able to satisfy

$$
\begin{array}{ll}
F(\bar{x})=V(\bar{x}) & \\
F_{i}(\bar{x})=V_{i}(\bar{x}) & \forall i=1, \ldots, n \\
F_{i j}(\bar{x})=V_{i j}(\bar{x}) & \forall i, j=1, \ldots, n .
\end{array}
$$

Thus, an FFF must have at least $1+n+1 / 2 n(n+1)$ parameters. Additional parsimony can be obtained by requiring the approximating function $F(x)$ to be homogeneous of degree one, which results in $n+1$ parametric restrictions (one linear constraint on first derivatives and $n$ linear constraints on second derivatives). Specifically, Euler's theorem here implies

$$
\begin{array}{ll}
\sum_{j=1}^{n} \bar{x}_{j} F_{j}(\bar{x})=F(\bar{x}) & \\
\sum_{j=1}^{n} \bar{x}_{j} F_{i j}(\bar{x})=0 & \forall j=1, \ldots, n .
\end{array}
$$

In general, such a linearly homogeneous function $F(x)$ is an FFF only if the true function $V(x)$ is also homogeneous of degree one. But below it is shown that such homogeneity restrictions are useful in a different setting.

Given this definition of flexibility, the following result is obtained. 
Proposition 1: FFF for a separable function. Suppose that a function $V(x)$ is separable in the partition $\hat{I}$, and define the function $F(x)$ to be separable in the same partition, i.e.,

$$
F(x) \equiv F^{0}\left(F^{1}\left(x^{1}\right), F^{2}\left(x^{2}\right), \ldots, F^{N}\left(x^{N}\right)\right)
$$

Then $F(x)$ is an FFF for $V(x)$ at $\bar{x}$ if:

(a) each $F^{r}(r=1,2, \ldots, N)$ is a general FFF for $V^{r}$ satisfying conditions (FLEX1) and (FLEX2) [but condition (FLEXO) need not be satisfied by any $F^{r}$ ], and

(b) $F^{0}$ is a FFF for a linear homogeneous function satisfying (FLEXO) and (FLEX2) under the constraint (HOMO2) [but condition (FLEXI) need not be satisfied by $F^{0}$ ].

The proof of this proposition is given in Appendix A. Essentially, Proposition 1 gives a procedure for constructing FFF for separable functions by carefully combining specific attributes of a general FFF and of a homogeneous FFF. But note that the "homogeneity" restrictions that Proposition 1 allows for the aggregator function $F^{0}$ are not predicated on the true aggregator function $V^{0}$ being homogeneous.

As a corollary, note that in multistage problems one can have additional partitions of each set $I^{r}$. For example, each $V^{r}$ may be written as $V^{r}\left(V^{r, 1}\left(x^{r, 1}\right), V^{r, 2}\left(x^{r, 2}\right), \ldots, V^{r, N_{r}}\left(x^{r, N_{r}}\right)\right)$. In this instance, if each $F^{r}$ has the same structure as the corresponding $V^{r}$, then a separable form $F$ will be an FFF for $V$ if each $F^{r, t}\left(x^{r, t}\right)$ has the same properties as the $F^{r}$ functions in Proposition 1, whereas $F^{0}$ and each $F^{r}$ only need to have the same properties as $F^{0}$ in Proposition 1. Essentially, this means that in a multistage separable form, an FFF can be obtained by constructing a form with the same structure, but with all the nesting functions (except the functions of the last stage) constrained to be homogeneous of degree one.

Proposition 1 applies to any functional forms $V(x)$ and $F(x)$ that have the assumed separable structure. Because in our application the true function $V($.$) will be a utility function, then further$ restrictions are allowed. Specifically, because a utility function (direct or indirect) is an ordinal function, then a linear transformation is always possible, so that without loss of generality we can invoke two additional restrictions. This observation produces an alternative representation for the 
sufficient conditions in Proposition 1, which is particularly useful for the multistage translog demand system to be derived later.

Proposition 2: FFF for a separable utility function. When $V(x)$ is a utility function, the function $F^{0}\left(F^{1}\left(x^{1}\right), F^{2}\left(x^{2}\right), \ldots, F^{N}\left(x^{N}\right)\right)$ is a FFF for $V^{0}\left(V^{1}\left(x^{1}\right), V^{2}\left(x^{2}\right), \ldots, V^{N}\left(x^{N}\right)\right)$ if:

(a') each $F^{r}$ is a general FFF for $V^{r}$ satisfying conditions (FLEX2) for all second derivatives and conditions (FLEXI) for all but one first derivative [condition (FLEXO) need not be satisfied by $F^{r}$ ], and

(b') $\quad F^{0}$ is an FFF for a linear homogeneous function satisfying (FLEX1) and (FLEX2) under the constraints (HOMO1) and (HOMO2) [condition (FLEXO) need not be satisfied].

\section{A Flexible and Separable Translog Demand System}

Propositions 1 and 2 offer a systematic avenue for modeling flexible separable structures. Adopting the translog specification of Christensen, Jorgenson and Lau (1975), we obtain the flexible and separable translog (FAST) demand model. Specifically, each price index $V^{r}\left(p^{r} / y\right)$ is written as

$$
\ln V^{r}\left(p^{r} / y\right)=\beta_{0}^{r}+\sum_{i \in I^{r}} \beta_{i} \log \left(p_{i} / y\right)+\frac{1}{2} \sum_{i \in I^{r}} \sum_{j \in I^{r}} \beta_{i j} \log \left(p_{i} / y\right) \log \left(p_{j} / y\right)
$$

whereas the aggregator function $V^{0}($.$) is written as$

$$
V^{0}(.)=-\left[\gamma_{0}+\sum_{r=1}^{N} \gamma_{r} \log V^{r}(.)+\frac{1}{2} \sum_{r=1}^{N} \sum_{s=1}^{N} \gamma_{r s} \log V^{r}(.) \log V^{s}(.)\right] .
$$

Note that the homogeneity property is satisfied by construction. Symmetry will be satisfied if $\beta_{i j}$ $=\beta_{j i}(\forall \mathrm{i}, \mathrm{j})$ and $\gamma_{r s}=\gamma_{s r}(\forall \mathrm{r}, \mathrm{s})$. Also, based on Propositions 1 and 2, without loss of generality we can set: 


$$
\begin{array}{ll}
\beta_{0}^{r}=0, & r=1,2, \ldots, N \\
\gamma_{0}=0 & \\
\sum_{i \in I^{r}} \beta_{i}=1, & r=1,2, \ldots, N \\
\sum_{r=1}^{N} \gamma_{r}=1 & \\
\sum_{S=1}^{N} \gamma_{r s}=0, & r=1,2, \ldots, N .
\end{array}
$$

In particular, the last set of restrictions follows from the fact that, from Propositions 1 and 2, we can restrict the first-stage aggregator function to be homogeneous.

Applying Roy's identity, the FAST share equations can be written as

$$
w_{i}=\frac{\left(\gamma_{r}+\sum_{s=1}^{N} \gamma_{r s} \log V^{s}\left(p^{s} / y\right)\right)\left(\beta_{i}+\sum_{j \in I^{r}} \beta_{i j} \log \left(p_{j} / y\right)\right)}{\sum_{g=1}^{N} B^{g}\left(p^{g} / y\right)\left(\gamma_{g}+\sum_{s=1}^{N} \gamma_{g s} \log V^{s}\left(p^{s} / y\right)\right)} i \in I^{r}
$$

where $w_{i} \equiv p_{i} q_{i} / y$ denotes the unconditional share of the $i^{\text {th }} \operatorname{good}, V^{s}\left(p^{s} / y\right)$ is the translog price function in (6), and the function $B^{g}\left(p^{g} / y\right)$ is defined as:

$$
B^{g}\left(p^{g} / y\right)=1+\sum_{j \in I^{g}} \sum_{i \in I^{g}} \beta_{i j} \log \left(p_{j} / y\right), \quad g=1,2, \ldots, N .
$$

For estimation purposes, however, it may be convenient to consider the conditional (withingroup) share equations $w_{i}^{r} \equiv p_{i} q_{i} / y_{r}$ and the group share equations $w^{r} \equiv y_{r} / y$. In view of equation (5) the conditional shares depend only on group prices and total expenditure, that is 


$$
w_{i}^{r}=\frac{\beta_{i}+\sum_{j \in I^{r}} \beta_{i j} \log \left(p_{j} / y\right)}{1+\sum_{k \in I^{r}} \sum_{j \in I^{r}} \beta_{k j} \log \left(p_{j} / y\right)} \quad \forall i \in I^{r}
$$

whereas the group share equations of our translog specification are:

$$
w^{r}=\frac{B^{r}\left(p^{r / y}\right)\left(\gamma_{r}+\sum_{s=1}^{N} \gamma_{r s} \log V^{s}\left(p^{s / y)}\right)\right.}{\sum_{g=1}^{N} B^{g}\left(p^{g} / y\right)\left(\gamma_{g}+\sum_{s=1}^{N} \gamma_{g s} \log V^{s}\left(p^{s / y)}\right)\right.} \quad r=1,2, \ldots, N
$$

To summarize, the model that has been constructed is both separable and flexible, while satisfying Barnett and Lee's (1985) "minimality property": i.e., FAST has the minimum number of parameters necessary to be an FFF while satisfying the property of indirect separability globally. It is interesting to note that this flexibility property is not always satisfied by previously used multistage demand systems; for example, the model of Blackorby, Boyce and Russell (1978) does not possess this property. ${ }^{8}$

\section{Asymmetric Separability}

The derivation of FAST so far implicitly assumes that there are at least two prices in each group. In some applications, however, it may be desirable to allow for asymmetric separability, such that a group may be composed by just one good. In such a degenerate case the derivation used above strictly does not apply, because for groups with only one price (say, $p_{i}$ ) identification of the conditional share equations requires $\beta_{i i}=0$ (in addition to the adding up restriction $\beta_{i}=$ 1). But one can easily rework Propositions 1 and 2 such that the first-stage function $F^{0}$ is not constrained to be homogeneous, thus freeing up one restriction for each of the second-stage groups. In terms of the FAST model, one can still use equations (6), (7), and (8), but with (8.5) replaced by the following: 


$$
\sum_{i \in I^{r}} \sum_{j \in I^{r}} \beta_{i j}=0, \quad r=1,2, \ldots, N
$$

\section{Curvature}

An integrable indirect utility function is quasiconvex in all normalized prices. As with the standard translog model, the FAST model developed here cannot satisfy this curvature condition globally. Thus, interest may center on checking whether the condition is satisfied at some relevant price-expenditure point (say, the mean point) by the estimated model. This can be done by checking that the Slutsky substitution matrix (equivalently, the matrix of elasticities of substitution) is negative semidefinite. Alternatively, one may be interested in maintaining the curvature condition at a point. For this purpose, it is desirable to take advantage of the multistage nature of the problem. Blackorby, Primont, and Russell (1978) show that, under indirect separability, the property of quasiconvexity of the indirect utility function is inherited by the aggregator function $V^{O}$ and by the reciprocals of the indices $V^{r}$. Whereas these curvature conditions are a necessary implication of separability, assuming quasiconvexity for $V^{0}$ and for the reciprocal of $V^{r}$ (for all $r$ ) is not sufficient to guarantee that the indirect utility function with the structure in (1) is itself quasiconvex. A sufficient condition is that the individual indices $V^{r}\left(p^{r} / y\right)$ are concave in their arguments and that the master utility function $V^{0}($.$) is quasiconvex in its$ arguments.

The curvature conditions on $V^{0}$ and $V^{r}$ can be maintained at a point by reparameterizing the

model in terms of the Cholesky decomposition (Moschini 1999). Appendix B explains in detail how curvature can be maintained locally in the FAST model. Again, the combination of these curvature assumptions is sufficient, although not necessary, to guarantee that the matrix of Allen elasticities of substitution is negative semidefinite at the point of interest. Note that the approach developed in Appendix B allows us to characterize the curvature properties of FAST in terms of the negative semidefiniteness of matrices of dimension $n_{r} \times n_{r}$ and $(N-1) \times(N-1)$. That is, for the FAST model the curvature property of the indirect utility function can be expressed in terms of the curvature of many aggregator functions of size potentially much smaller than that of the general model. Because the Cholesky decomposition used to maintain the curvature conditions 
becomes increasingly complex as the number of terms increases, the FAST model proposed here offers a considerable simplification relative to the general model for the purpose of maintaining the theoretical curvature properties.

\section{An Application to Canadian Food Demand}

To illustrate the FAST demand system just derived, the results of a 10-good complete demand system emphasizing food consumption are presented. Specifically, 7 of the 10 specified goods represent food consumed at home by Canadians. The 10 consumption categories are: $q_{1}$ $=$ beef and pork; $q_{2}=$ poultry and fish; $q_{3}=$ dairy products; $q_{4}=$ fruits; $q_{5}=$ vegetables; $q_{6}=$ bread and bakery; $q_{7}=$ other food; $q_{8}=$ nonalcoholic beverages; $q_{9}=$ food away from home; and, $q_{10}=$ nonfood. Admittedly, the extreme aggregation assumption about the nonfood sector is very restrictive, but when interest centers on food demand the resulting complete system is still preferable to a system that ignores the nonfood sector (such as a second-stage demand system conditional on food expenditures).

Data on consumption and prices are available for Canada in terms of annual food expenditures from the system of national accounts of Statistics Canada. This data set provides both current and constant price consumer expenditures for fairly disaggregated food categories consumed at home. These food expenditure data were supplemented with corresponding food away from home data, and nonfood expenditure data, from Agriculture Canada's Handbook of Food Expenditures, Prices and Consumption. Implicit price indices for each category were constructed by dividing current expenditures by constant-price expenditures. The entire data set used covers the period 1961-1988. Before estimation, all prices and total per-capita expenditure were normalized by dividing through by the respective (geometric) mean. More details on the data are available from the author upon request.

It is postulated that food consumed at home is indirectly separable from the consumption of other goods. Specifically, the indirect utility function that generates the demand system is written as

$$
V=V^{0}\left(V^{1}\left(p_{1} / y, p_{2} / y, \ldots, p_{7} / y\right), V^{2}\left(p_{8} / y, p_{9} / y, p_{10} / y\right)\right)
$$


In addition to the FAST model entailed by the indirect utility function in (13), for comparison purposes the (nonseparable) basic translog (BTL) model was also estimated. The BTL share system is written as:

$$
w_{i}=\frac{\alpha_{i}+\sum_{j=1}^{n} \alpha_{i j} \log \left(p_{j} / y\right)}{1+\sum_{k=1}^{n} \sum_{j=1}^{n} \alpha_{k j} \log \left(p_{j} / y\right)} \quad i=1,2, \ldots, n
$$

where $\alpha_{i j}=\alpha_{j i}(\forall \mathrm{i}, \mathrm{j})$ and $\Sigma_{i} \alpha_{i}=1$. Note that the BTL model has 64 parameters to be estimated, whereas the FAST model has 44 parameters. $^{9}$

Because of the motivation discussed in section 2, it is also of interest to compare IWS with DWS. Unfortunately, it is not straightforward to compare DWS and IWS based on unconditional demand systems because, as explained earlier, DWS is not amenable to yielding a flexible complete demand system in close form. Thus, in what follows, the two conditional demand systems obtained from the assumed IWS structure of equation (13) are compared with the two conditional demand systems produced by DWS in the same partition, namely the two conditional systems that would result if the direct utility function were:

$$
U=U^{0}\left(U^{1}\left(q_{1}, q_{2}, \ldots, q_{7}\right), U^{2}\left(q_{8}, q_{9}, q_{10}\right)\right)
$$

As discussed earlier, the conditional demand systems implied by IWS will have the form of equation (11). But for the conditional demand system obtained under the DWS structure of equation (14) the relevant conditioning variable is $y_{r}$ (instead of $y$ ). The translog form for such a conditional system is:

$$
w_{i}^{r}=\frac{\tilde{\beta}_{i}+\sum_{j \in I^{r}} \tilde{\beta}_{i j} \log \left(p_{j} / y_{r}\right)}{1+\sum_{k \in I^{r}} \sum_{j \in I^{r}} \tilde{\beta}_{k j} \log \left(p_{j} / y_{r}\right)} \quad \forall i \in I^{r}, r=1,2
$$




\section{Estimation}

Estimation of the BTL model and of the conditional demand systems can follow standard practice for seemingly unrelated regression systems. As for FAST, two main estimation strategies are possible. Perhaps the most natural procedure is to estimate $\left(n_{r}-1\right)$ conditional share equations (11) for each group, plus $(N-1)$ group share equations (12), for a total of $(n-1)$ equations. For such an approach, the recursive structure of IWS is amenable to an appealing internally consistent stochastic specification which can be implemented either sequentially or simultaneously. Sequential estimation entails a two-step procedure: first, estimate the within-group share systems, independently for each of the $N$ groups; second, taking the estimated $\left(\beta_{i}, \beta_{i j}\right)$ as given, compute the indices $\log V^{r}$ and $B^{r}$ and estimate the $(N-1)$ group share equations conditional on these indices. This two-step procedure is computationally attractive because it will typically entail estimation of relatively small systems. The possible drawback of this two-step procedure is that, in the second step, group shares are estimated conditional on the estimated parameters of the first stage (which, strictly, are random variables). ${ }^{10}$ This problem, of course, is not present if the $\left(n_{r}\right.$ 1) conditional share equations (11) and the ( $N$ - 1) group share equations (12) are estimated simultaneously.

The alternative strategy for FAST is to estimate $(n-1)$ unconditional shares as in equation (9), similar to the standard approach of estimating demand systems. In the application that follows, this latter procedure is adopted because of the desire to compare the FAST model with the (nonseparable) BTL model (a comparison that is greatly simplified if we have the same lefthand-side variables and the same stochastic assumptions). The stochastic system of demand equations that are estimated can be written as standard systems ofs eemingly unrelated regressions:

$$
z_{t}=f\left(\mu, X_{t}\right)+v_{t} \quad t=1,2, \ldots, T,
$$

where $z_{t}$ is a vector of $M$ shares at time $t,{ }^{11} \mu$ is the vector of all coefficients to be estimated, $X_{t}$ is the vector of the corresponding exogenous variables at time $t$, and $v_{t}$ is a vector of $M$ error terms. To allow the random terms to display serial correlation, we write $v_{t}=\rho v_{t-1}+e_{t}$. Note that the autocorrelation coefficient is constrained to be the same for all equations, which provides the 
simplest structure guaranteeing that the resulting stochastic system satisfies adding-up (Berndt and Savin 1975; Moschini and Moro 1994). The error terms $e_{t}$ satisfy $E\left[e_{t}\right]=0$ and are serially uncorrelated but contemporaneously correlated; in other words, $E\left[e_{t} e_{t}^{\prime}\right]=\Omega(\forall t)$, and $E\left[e_{t} e_{s}^{\prime}\right]=$ $0(\forall t \neq s)$. The additional assumption that the $e_{t}$ 's are multinormally distributed leads to maximum likelihood estimation (e.g., Davidson and MacKinnon 1993, chapter 9), which is invariant with respect to which equation is omitted.

\section{Results}

It should be noted at this point that all the models that are to be compared are nonnested. Furthermore, the hypothesis of IWS separability was motivated as a coherent strategy to reduce the parametric burden of estimating complete demand systems (rather than being more compelling than DWS on behavioral grounds, say). Hence, attempting formal hypothesis testing may be too ambitious a task in our setting. Given that, and in light of related considerations articulated by Granger, King and White (1995), model selection criteria are used to illuminate the performance of the various models that were estimated. Specifically, the comparison of models relies on Schwarz's (1978) criterion, a rule that is firmly rooted in information theory and that has good asymptotic properties. For the system of equations setting used here, the Schwarz criterion favors the model which minimizes:

$$
S C=-\mathscr{L}(\hat{\mu})+\frac{K}{2} \log (M T)
$$

where $\mathscr{L}(\hat{\mu})$ is the maximized $\log$ likelihood value of the model, $K$ is the number of parameters in the model, and $(M T)$ is the effective number of observations.

Results for the various models that were estimated are reported in Table 1. It appears that, based on the SC model selection criterion, the FAST model dominates the (nonseparable) BTL model. As for the comparison between IWS and DWS, because the conditional systems derived under either notion of separability have the same number of parameters, the SC in these cases reduces to comparing likelihood values. From the results of Table 1 it appears that IWS is superior to DWS for the first conditional system (food at home), and essentially equivalent to 
DWS for the second conditional system (all other goods). ${ }^{12}$ Overall, one can conclude that the particular data set at hand offers some support for the postulated indirect separability structure and for the proposed FAST specification.

The main payoff of the FAST modeling strategy is the estimation, in a relatively parsimonious model, of a complete matrix of unconditional elasticities. Such unconditional Marshallian price and expenditure elasticities for the FAST model, estimated at the mean point, are reported in Table 2. It is worth noting that the estimated FAST model turns out to satisfy the curvature conditions at the mean point, whereas the BTL model does not (for BTL, one of the nine nonzero eigenvalues is positive). The model shows that demand for food consumed at home is very inelastic with respect to total per-capita expenditure $y$. The (weighted) average of expenditure elasticities for food consumed at home (the first seven goods) is about 0.12 . This finding is consistent with typical consumption patterns of developed countries, where food consumption constitutes a relatively small and decreasing component of expenditure allocation. Food consumed away from home and the aggregate of all other goods, on the other hand, turn out to have elastic demand (luxury goods).

Demands for food items are all inelastic with respect to own price, with the exception of the poultry and fish group. This result is of some interest, because in Canada the poultry market has been constrained by restrictive supply management policies exercised by producer marketing boards. As a result, Canadian poultry prices are much higher and per-capita consumption is much smaller than in the United States, and the concern has been repeatedly expressed that such policies may have moved market equilibrium conditions closer to that of a monopoly situation. Finding that poultry demand is price-elastic is certainly consistent with that scenario.

Marshallian cross elasticities in Table 2 are clearly affected by the postulated indirect separability structure. In particular, if $\varepsilon_{i k} \equiv \partial \log q_{i} / \partial \log p_{k}$ denotes Marshallian cross-price elasticities, then when one considers goods belonging to different partitions under IWS such Marshallian elasticities must satisfy:

$$
\varepsilon_{i k}=\varepsilon_{j k} \quad \forall(i, j) \in I^{r} \text { and } k \notin I^{r} \text {. }
$$


But Table 2 perhaps overstates the straightjacket brought about by the separability assumption because, as Blackorby and Russell (1989) convincingly argue, substitutability in the many goods case is best illustrated by Morishima elasticities. Such elasticities are not affected in an obvious way by the assumption of separability. Table 3 reports the Morishima elasticities at the mean point for the estimated model. Virtually all such elasticities are positive, indicating that substitution relations are pervasive in this estimated model.

\section{Conclusion}

This article has presented a new demand model that exploits the notion of indirect separability to derive a multistage demand system. The chief advantage of this approach is that separability of the indirect utility function allows a straightforward derivation of a consistent parameterization of demand relations at various budgeting stages.The article also offers two propositions that characterize flexible and separable functional forms, which provide a systematic procedure to specify a demand model that fulfills the requirement of flexibility while satisfying separability globally. The implementation of the proposed approach relies on the translog functional form to derive the FAST demand system. Such a specification seems particularly attractive for the purpose of modeling large complete demand systems. The model was illustrated with an application to Canadian food demand. The FAST model was superior to the nonseparable BTL model based on model selection criteria. Overall, it seems that IWS may be a promising alternative to DWS when the focus is to specify and estimate a complete demand system, and that the particular FAST parameterization presented in this article may work well for that purpose. 
Table 1. Summary of estimation results, all models

Model

Number of Maximized

Schwarz

parameters log-likelihood

criterion

Full systems:

FAST

44

1607.6

$-1491.1$

Basic Translog

64

1636.0

$-1481.1$

Conditional systems 1 (food at home):

IWS

34

764.6

DWS

34

754.6

Conditional systems 2 (other goods):

IWS

DWS
8

8
327.6

326.9 
Table 2. Estimated Marshallian elasticities at the mean point, FAST model

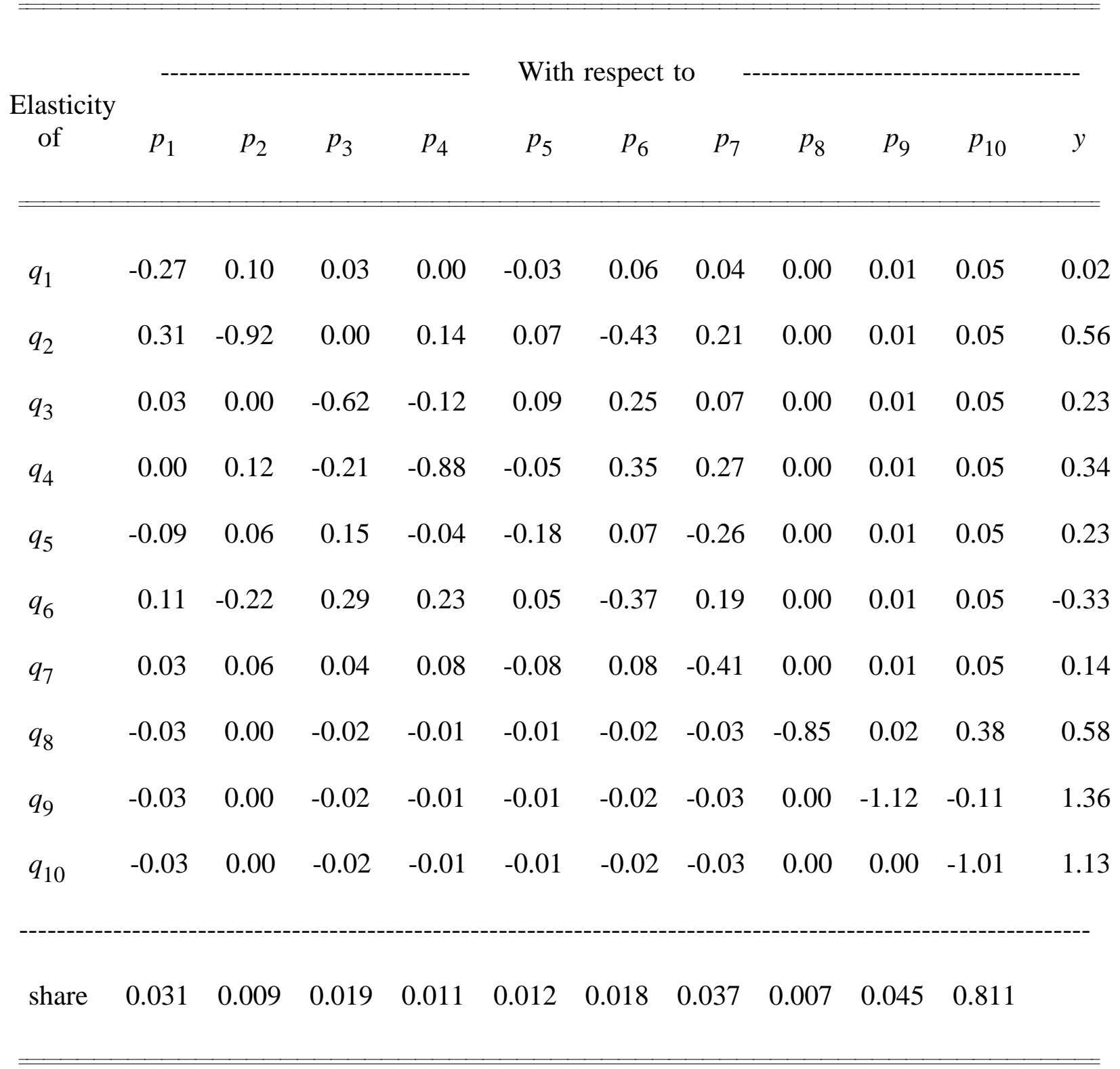


Table 3. Estimated Morishima elasticities of substitution at the mean point, FAST model

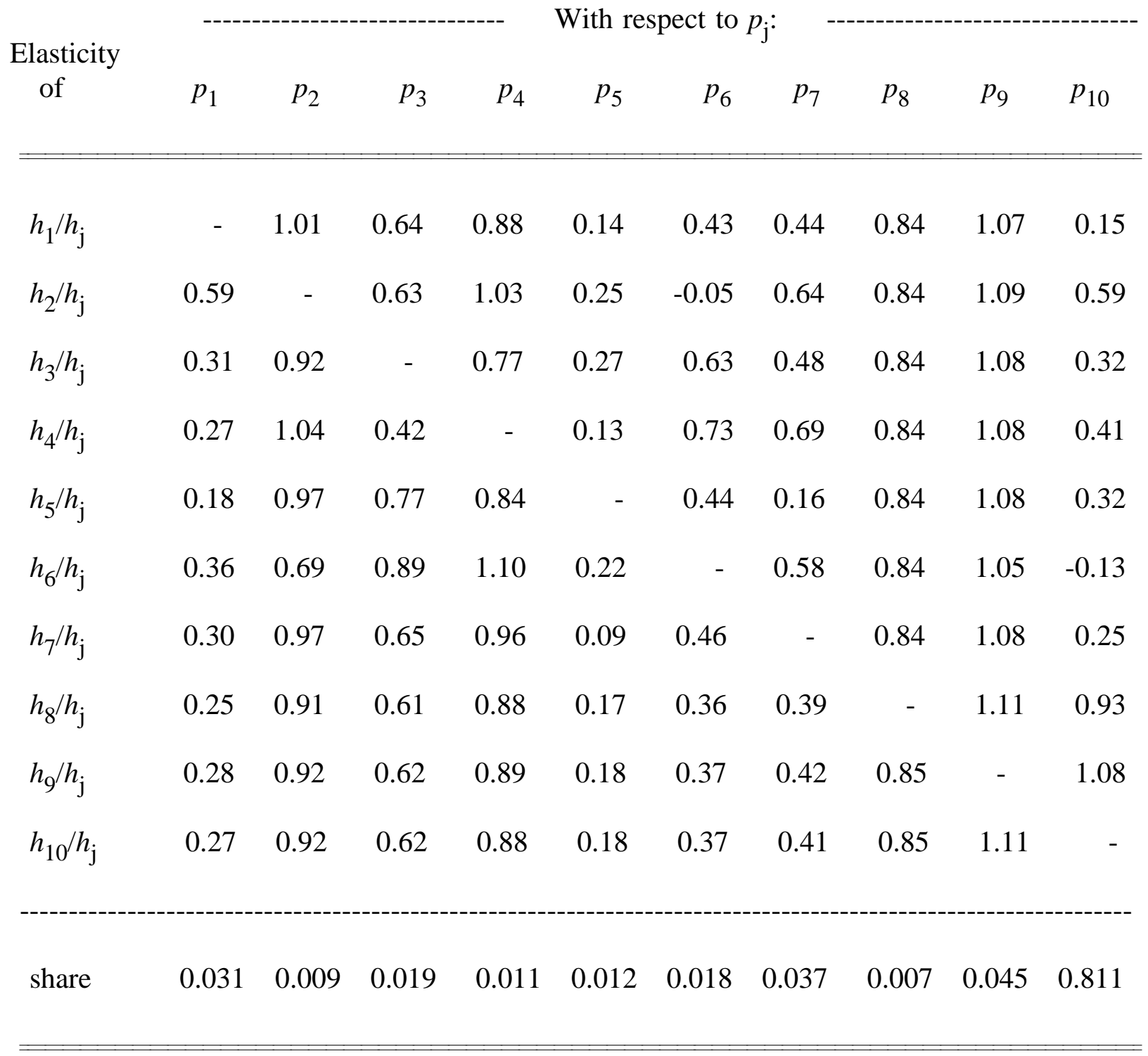

Note: $h_{\mathrm{j}}$ denotes the Hicksian demand function. 
A Flexible Multistage Demand System / 23

\section{Endnotes}

1. An alternative that puts a more amorphous structure on consumer preferences is the specification of "semiflexible" functional forms (Diewert and Wales 1988; Moschini 1998).

2. Yet others have postulated DWS with each subutility function having a dual representation that is of the Gorman polar form (e.g., Michalek and Keyzer 1992). Such quasi-homothetic subutility functions entail that the first-stage of the budgeting process depends on two price indices per group.

3. For example, the approximation used by Edgerton (1997) fails to ensure the Slutsky symmetry property of unconditional demand functions (Carpentier and Guyomard 2001).

4. Alternatively, one could consider higher-order approximations of the utility function (Hayes 1988), which may however result in an unmanageable parametric burden for large demand systems.

5. As shown by Gorman (1959), for the first-stage problem to depend only on one price index per group one needs the perfect price aggregation conditions mentioned in the introduction.

6. Here we are using the same partition for DWS and IWS. But of course the functions $V^{r}($.) are not dual to $U^{r}$ (.) (IWS is not equivalent to DWS unless the group utility functions are homothetic).

7. For the same partition $\hat{I}$ DWS and IWS entail the same number of restrictions on elasticities, namely $1 / 2\left[n(n-1)-\Sigma_{r} n_{r}\left(n_{r}-1\right)-N(N-1)\right]$, where $n_{r}$ denotes the number of goods in the $r^{\text {th }}$ group. 8. In particular, for two-stage models, the generalized S-branch model of Blackorby, Boyce and Russell (1978) has more parameters than necessary to meet the definition of flexibility when the number of groups does not exceed five, whereas the model does not satisfy the definition of flexibility when the number of groups is larger than five.

9. Thus, the assumed separable structure yields a parametric burden roughly consistent with the rule of thumb cited by Chalfant and Gallant (1985), who noted that for $n$ goods and $T$ observations the number of parameters should not exceed $[(n-1) T]^{2 / 3}$.

10. When this two-step procedure is adopted, it seems that the FAST parameterization involving $\left(8.5^{\prime}\right)$ rather than (8.5) performs better, in the sense that the estimates from the recursive two-step procedure are much closer to those one obtains from estimating all equations simultaneously 
(given the same stochastic assumptions).

11. For example, for the unconditional share models (FAST and BTL) we have $M=9$ (one of the shares is omitted because of the known singularity problem of share equation systems).

12. Because the focus here is not on the estimated parameters, I do not account for the fact that $y_{r}$ in the conditional demand systems derived from DWS is endogenous (which is itself a major criticism of many empirical applications that appeal to DWS to limit the analysis to second-stage demand functions). Blundell (1988) discusses a simple econometric procedure that may be used to correct for expenditure endogeneity; see also LaFrance (1991) and Edgerton (1993). 


\section{Appendix A. Proofs of Propositions 1 and 2}

\section{Proof of Proposition 1}

Given the assumed separable structures for both $V$ and $F$, for flexibility we need to satisfy

$$
\begin{aligned}
& V^{0}=F^{0} \\
& V_{r}^{0} V_{i}^{r}=F_{r}^{0} F_{i}^{r} \quad i \in I^{r}, \forall r \\
& V_{r r}^{0} V_{i}^{r} V_{j}^{r}+V_{r}^{0} V_{i j}^{r}=F_{r r}^{0} F_{i}^{r} F_{j}^{r}+F_{r}^{0} F_{i j}^{r} \quad \forall i, j \in I^{r}, \forall r \\
& V_{r s}^{0} V_{i}^{r} V_{k}^{s}=F_{r s}^{0} F_{i}^{r} F_{k}^{s} \\
& \forall i \in I^{r} \text { and } k \in I^{s}, \forall r \neq s,
\end{aligned}
$$

where all functions are evaluated at the point $\bar{x}$. These conditions can be rewritten as

$$
\begin{aligned}
& V^{0}=F^{0} \\
& \frac{V_{r}^{0} V_{i}^{r}}{F_{r}^{0}}=F_{i}^{r} \quad i \in I^{r}, \forall r \\
& \frac{V_{r r}^{0} V_{i}^{r} V_{j}^{r}+V_{r}^{0} V_{i j}^{r}-F_{r r}^{0} F_{i}^{r} F_{j}^{r}}{F_{r}^{0}}=F_{i j}^{r} \quad \forall i, j \in I^{r}, \quad \forall r \\
& \frac{V_{r s}^{0} F_{r}^{0} F_{s}^{0}}{V_{r}^{0} V_{s}^{0}}=F_{r s}^{0} \quad \forall r \neq s,
\end{aligned}
$$

where (A.4) uses (A.2). Condition (A.1) will be satisfied if $F^{0}$ satisfies (FLEX0). Condition (A.2) will be satisfied if each $F^{r}$ satisfies (FLEX1). Condition (A.3) will be satisfied if each $F^{r}$ satisfies (FLEX2). Finally, condition (A.4) will be satisfied if $F_{r s}^{0}(r \neq s)$ can attain an arbitrary value. Note that, because the terms $F_{r r}^{0}$ do not need to attain arbitrary values, we can set $\Sigma_{s} \kappa_{s}$ $F_{r s}^{O}=O(\forall \mathrm{r})$ for some constants $\kappa_{s}$ (i.e., $F^{0}$ can be a linearly homogeneous FFF). Finally, in the 
conditions above, $F_{r}^{O}$ can take any (nonzero) value (thus, the aggregator functions $F^{0}$ need not satisfy (FLEX1)].

Proof of Proposition 2

Ordinality of utility implies that, if $V^{A}$ is a utility function describing a given set of preferences, then $V \equiv \kappa_{0}+\kappa_{l} V^{A}$, where $\kappa_{0}$ and $\kappa_{1}>0$ are arbitrary constants, represents the same preferences. Because $\kappa_{0}$ is arbitrary, a FFF for a utility function does not need to satisfy (FLEX0); thus, (A.1) does not need to be satisfied. Next note that in satisfying (A.2), $F_{r}^{0}$ can substitute for one $F_{i}^{r}$; however, because $F^{0}$ is assumed homogeneous of degree one, only ( $N$ 1) free $F_{r}^{0}$ are available. But that is all that is required to prove Proposition 2 (given Proposition 1) because, given that $\kappa_{1}$ is arbitrary, marginal utilities can be subject to one linear restriction. 
A Flexible Multistage Demand System / 27

\section{Appendix B. Local Curvature Conditions for the FAST Model}

Consider first the concavity of indices $V^{r}$. This condition requires that the Hessian of each price function $V^{r}$ be negative semidefinite. The elements of this Hessian can be found by differentiating (6); for that, the following identity may be useful:

$$
\frac{\partial^{2} V^{r}}{\partial p_{i} \partial p_{j}} \equiv \frac{V^{r}}{p_{i} p_{j}}\left[\frac{\partial^{2} \log V^{r}}{\partial \log p_{i} \partial \log p_{j}}+\frac{\partial \log V^{r}}{\partial \log p_{i}} \frac{\partial \log V^{r}}{\partial \log p_{j}}-\delta_{i j} \frac{\partial \log V^{r}}{\partial \log p_{i}}\right]
$$

where $\delta_{i j}$ is the Kronecker delta. From (6) and (B.1) it is clear that the translog form assumed here cannot satisfy the required curvature property globally (that is, for all levels of normalized prices). The curvature property can be verified or maintained locally, say for a relevant range of prices [as in the Bayesian approach analyzed by Terrell (1996)] or for a representative point in the normalized price space. Here the latter approach is outlined. Without loss of generality, let the point of interest be $p=1$. Then at this point:

$$
\frac{\partial^{2} V^{r}}{\partial p_{i} \partial p_{j}}=\beta_{i j}+\beta_{i} \beta_{j}-\delta_{i j} \beta_{i} \quad(i, j) \in I^{r}
$$

Defining the matrix $B \equiv\left[\beta_{i j}\right]$, the vector $b \equiv\left[\beta_{i}\right]$, and the matrix $\hat{b} \equiv \operatorname{diag}(b)$, then local concavity of the price index $V^{r}$ requires that the matrix $\left[B+b b^{\prime}-\hat{b}\right]$ be negative semidefinite. This condition can be easily checked upon obtaining the estimated parameters. Alternatively, it can be maintained by using the Cholesky decomposition as suggested by Lau (1978). According to this decomposition, in the form used by Diewert and Wales (1987), a sufficient condition for a matrix $G$ to be positive semidefinite is that it can be written as $G=A A^{\prime}$, where $A \equiv\left[a_{i j}\right]$ is a lower triangular matrix. Hence, the desired curvature property can be maintained at a point by setting $B=-b b^{\prime}+\hat{b}-A A^{\prime}$, which essentially entails rewriting the $\beta_{i j}$ in terms of the $\left\{\beta_{i}, a_{i j}\right\}$ parameters.

Now consider the matter of the quasiconvexity of $V^{0}$ (or, equivalently, quasiconcavity of the reciprocal of $V^{0}$ ). Rather than deal directly with this curvature condition, it is more convenient 
(and fully equivalent) to characterize curvature in terms of the concavity of the dual expenditure function. Because our interest, at this juncture, centers on the curvature properties of $V^{0}$ in the price indices $V^{r}$, we may note that such curvature properties should hold regardless of the shape of the price indices $V^{r}$ in $p^{r}$. Hence, to characterize the curvature of $V^{0}$ in $V^{r}$ it is convenient to consider the case where the price indices satisfy $V^{r}\left(p^{r} / y\right)=\pi^{r}\left(p^{r}\right) / y$. (This means that the $V^{r}$ themselves are linear homogeneous in normalized prices, implying that the separability structure being considered reduces to homothetic separability such that IWS is equivalent to DWS. But it needs to be emphasized again, however, that there is no loss of generality to consider homothetic separability at this juncture because we are investigating the curvature properties of $V^{0}$ in $V^{r}$.) Given the indices $\pi^{r}$, the expenditure function dual to $V^{O}(\pi / y)$ can be written as $C(\pi, u)$, where $\pi \equiv\left[\pi^{l}, \ldots, \pi^{N}\right]$ is the vector of price indices $\pi^{r}$. Twice differentiating the identity $V^{0}(\pi / C(\pi, u))$ $\equiv u$ yields

$$
\frac{\partial^{2} V^{0}}{\partial \pi^{r} \partial \pi^{s}}+\frac{\partial^{2} V^{0}}{\partial y \partial \pi^{s}} \frac{\partial C}{\partial \pi^{r}}+\frac{\partial^{2} V^{0}}{\partial \pi^{r} \partial y} \frac{\partial C}{\partial \pi^{s}}+\frac{\partial^{2} V^{0}}{\partial y^{2}} \frac{\partial C}{\partial \pi^{r}} \frac{\partial C}{\partial \pi^{s}}+\frac{\partial V^{0}}{\partial y} \frac{\partial^{2} C}{\partial \pi^{r} \partial \pi^{s}}=0 .
$$

Recalling that $\left(\partial C / \partial \pi^{r}\right)=-\left(\partial V^{0} \partial \pi^{\gamma}\right)\left(\partial V^{0} \partial y\right)^{-1}$ by Roy's identity, then by (B.2) one can express the Hessian of the cost function (which must be negative semidefinite because of the concavity of $C(\pi, u)$ in $\pi)$, in terms of first and second derivatives of the indirect utility function. Specifically, differentiating $V^{0}$ in equation (7), and evaluating the derivatives at the point $p=$ $y=1$, we obtain

$$
\frac{\partial^{2} C(1,1)}{\partial \pi^{r} \partial \pi^{s}} \equiv \Gamma_{r s}=\gamma_{r s}-\gamma_{r} \theta_{s}-\gamma_{s} \theta_{r}+\gamma_{r} \gamma_{s}\left(1+\sum_{g=1}^{N} \theta_{g}\right)-\delta_{r s} \gamma_{s}
$$

where $\delta_{r s}$ is the Kronecker delta, and where the following reparameterization proves useful for translog specifications:

$$
\theta_{r} \equiv \sum_{s=1}^{N} \gamma_{r s}
$$


The curvature condition at the point $\pi^{r}=y=1$, therefore, is that the matrix whose elements are in (B.3) is negative semidefinite. Such a condition can be maintained by rewriting the righthand side of (B.3) in terms of the Cholesky decomposition introduced earlier. Because the cost function is homogeneous of degree plus one in prices, the Hessian of the cost function is singular. In other words, $\Sigma_{s} \Gamma_{r s}=0(\forall r)$. Thus, one of the eigenvalues will by necessity be zero. Hence, the desired curvature condition can be maintained by setting $\Gamma=-A A^{\prime}$, where $\Gamma$ is the $(N-1) \times(N-1)$ sub-Hessian matrix whose elements are in (B.3), and $A$ here is a $(N-1) \times(N-1)$ lower triangular matrix. This essentially entails writing the $\gamma_{r s}$ parameters in terms of the $\left\{\gamma_{r}, a_{r s}\right\}$ parameters.

Parameters $\theta_{r}$ are, to this point, unconstrained. But, when the second stage does not include degenerate aggregates (that is, groups have at least two goods each), then, as argued earlier, the function $V^{0}$ can be approximated by a linearly homogeneous function without destroying the flexibility of the model. In other words, we can set $\theta_{r}=0$ for all $r$, which greatly simplifies the relevant Hessian terms in (B.3), which can then be written as

$$
\Gamma_{r s}=\gamma_{r s}+\gamma_{r} \gamma_{s}-\delta_{r s} \gamma_{r}
$$

Thus, in this instance the restrictions of quasiconvexity of $V^{0}$ turn out to be the same as requiring that $V^{0}$ be convex. This is to be expected because, as shown by Newman (1969), a positive and linearly homogeneous function that is quasiconcave is also concave. 


\section{Appendix C. Elasticities for the FAST Demand System}

Because Marshallian elasticities satisfy

$$
\begin{aligned}
& \varepsilon_{i j}=\frac{1}{w_{i}} \frac{\partial w_{i}}{\partial \log \left(p_{j}\right)}-\delta_{i j} \\
& \varepsilon_{i}=\frac{1}{w_{i}} \frac{\partial w_{i}}{\partial \log (y)}+1,
\end{aligned}
$$

where $\delta_{i j}$ is the Kronecker delta $\left(\delta_{i j}=1\right.$ if $i=j$, and $\delta_{i j}=0$ otherwise), by using the structure of the FAST share equations in the text, it follows that

$$
\begin{aligned}
& \varepsilon_{i j}=\frac{1}{D}\left[\frac{\Gamma^{r} \beta_{i j}+\gamma_{r r} B_{i} B_{j}}{w_{i}}-\sum_{g=1}^{N} \gamma_{g r} B_{j} B^{g}-\Gamma^{r}\left(\sum_{m \in I^{r}} \beta_{j m}\right)\right]-\delta_{i j}, \quad(i, j) \in I^{r} \\
& \varepsilon_{i k}=\frac{1}{D}\left[\frac{\gamma_{r s} B_{k} B_{i}}{w_{i}}-\sum_{g=1}^{N} \gamma_{g s} B_{k} B^{g}-\Gamma^{s}\left(\sum_{m \in I^{s}} \beta_{k m}\right)\right], \quad i \in I^{r} \text { and } k \in I^{s} \\
& \varepsilon_{i}=1-\frac{1}{D}\left[\frac{\Gamma^{r}\left(\sum_{j \in I^{r}} \beta_{i j}\right)+B_{i} \sum_{s=1}^{N} \gamma_{s r} B^{s}}{w_{i}}-\sum_{g=1}^{N} \sum_{s=1}^{N} \gamma_{s g} B^{s} B^{g}-\sum_{s=1}^{N} \Gamma^{s}\left(\sum_{k \in I^{s}} \sum_{m \in I^{s}} \beta_{k m}\right)\right], \quad i \in I^{r}
\end{aligned}
$$

where $D$ is the denominator of the right-hand side of equation (9), $B^{g}$ is defined in equation (10), and $B_{i}$ and $\Gamma^{r}$ are defined as follows:

$$
B_{i} \equiv \beta_{i}+\sum_{j \in I^{r}} \beta_{i j} \log \left(p_{j} / y\right) \quad i \in I^{r}
$$




$$
\Gamma^{r} \equiv \gamma_{r}+\sum_{s=1}^{N} \gamma_{r s} \log V^{s}
$$

If the data are normalized such that the point of interest is $p_{i}=y=1(\forall i)$, then at this point $w_{i}=$ $\gamma_{r} \beta_{i}, D=1$, and

$$
\begin{aligned}
& \varepsilon_{i j}=\frac{\beta_{i j}}{\beta_{i}}+\frac{\gamma_{r r} \beta_{j}}{\gamma_{r}}-\gamma_{r}\left(\sum_{m \in I^{r}} \beta_{j m}\right)-\delta_{i j} \quad(i, j) \in I^{r} \\
& \varepsilon_{i k}=\frac{\gamma_{r s} \beta_{k}}{\gamma_{r}}-\gamma_{s}\left(\sum_{m \in I^{s}} \beta_{k m}\right) \quad i \in I^{r} \quad \text { and } \quad k \in I^{s} \\
& \varepsilon_{i}=1-\frac{\sum_{j \in I^{r}} \beta_{i j}}{\beta_{i}}+\sum_{s=1}^{N} \gamma_{s}\left(\sum_{k \in I^{s}} \sum_{m \in I^{s}} \beta_{k m}\right) \quad i \in I^{r} .
\end{aligned}
$$

If $\left(8.5^{\prime}\right)$ is used instead of (8.5), then the formula for income elasticities simplifies further to:

$$
\varepsilon_{i}=1-\frac{\sum_{j \in I^{r}} \beta_{i j}}{\beta_{i}} \quad i \in I^{r}
$$

Also, formulae for compensated (Hicksian) elasticities $\eta_{i j}$ and Allen-Uzawa substitution elasticities $\sigma_{i j}$ can be found readily by noting that, by the Slutsky equation, $\eta_{i j}=\varepsilon_{i j}+w_{j} \varepsilon_{i}$ and $\sigma_{i j}=\varepsilon_{i j} / w_{j}+$ $\varepsilon_{i}$ (and recalling that, at the point of interest, $w_{i}=\gamma_{r} \beta_{i}$ ). Finally, the Morishima elasticities of substitution reported in Table 3 satisfy $\sigma_{i j}^{M}=\eta_{i j}-\eta_{j j}$ 


\section{References}

Aizcorbe, Ana M. 1992. A note on empirical tests of separability and the approximation view of functional forms. Southern Economic Journal 59: 310-318.

Anderson, Ronald W. 1979. Perfect price aggregation and empirical demand analysis. Econometrica 47: 1209-1230.

Barnett, William A., and Yul W. Lee. 1985. The global properties of the Minflex Laurent, Generalized Leontief, and Translog flexible functional forms. Econometrica 53: 1421-1437.

Barr, D.G., and K. Cuthbertson. 1994. The demand for financial assets held in the U.K. by the overseas sector: An application of two-stage budgeting. The Manchester School of Economic and Social Studies 62: 1-20.

Berndt, Ernst R., and N. Eugene Savin. 1975. Estimation and hypothesis testing in singular equation systems with autoregressive disturbances. Econometrica 43: 937-957.

Blackorby, Charles, Richard Boyce, and R. Robert Russell. 1978. Estimation of demand systems generated by the Gorman Polar Form: A generalization of the S-Branch Utility Tree. Econometrica 46: $345-362$.

Blackorby, Charles, Daniel Primont, and R. Robert Russell. 1977. On testing separability restrictions with flexible functional forms. Journal of Econometrics 5: 195-209.

Blackorby, Charles, Daniel Primont, and R. Robert Russell. 1978. Duality, Separability, and Functional Structure: Theory and Economic Applications. New York, NY: North-Holland.

Blackorby, Charles, Daniel Primont, and R. Robert Russell. 1998. Separability: A survey. In Handbook of Utility Theory, Volume 1, edited by S. Barberà, P.J. Hammond, and C. Seidl. Dordrecht: Kluwer Academic Publishers.

Blackorby, Charles, and R. Robert Russell. 1989. Will the real elasticity of substitution please stand up? American Economic Review 79: 882-888.

Blundell, Richard. 1988. Consumer behavior: Theory and empirical evidence -- A survey. Economic Journal 98: 16-65.

Brown, Murray, and Dale M. Heien. 1972. The S-Branch Utility Tree: A generalization of the Linear Expenditure System. Econometrica 20: 737-747.

Browning, Martin, and Costas Meghir. 1991. The effects of male and female labor supply on commodity demands. Econometrica 59: 925-951. 
Carpentier, Alain, and Hervé Guyomard. 2001. Unconditional elasticities in two-stage demand systems. American Journal of Agricultural Economics, 83 (in press).

Chalfant, James A., and A. Ronald Gallant. 1985. Estimating substitution elasticities with the Fourier cost function. Journal of Econometrics 28: 205-222.

Christensen, Laurits R., Dale W. Jorgenson, and Lawrence J. Lau. 1975. Transcendental logarithmic utility functions. American Economic Review 65: 367-383.

Davidson, Russell, and James G. MacKinnon. 1993. Estimation and inference in econometrics. Oxford: Oxford University Press.

Denny, Michael, and Melvyn Fuss. 1977. The use of approximation analysis to test for separability and the existence of consistent aggregates. American Economic Review 67: 404-418.

Diewert, W. Erwin. 1974. Applications of Duality Theory. In Frontiers of quantitative economics, volume II, edited by M.D. Intriligator and D.A. Kendrick. Amsterdam: North-Holland.

Diewert, W. Erwin, and Terence J. Wales. 1987. Flexible functional forms and global curvature conditions. Econometrica 55: 43-68.

Diewert, W. Erwin, and Terence J. Wales. 1988. A normalized quadratic semiflexible functional form. Journal of Econometrics 37: 327-342.

Diewert, W. Erwin, and Terence J. Wales. 1995. Flexible functional forms and tests of homogeneous separability. Journal of Econometrics 67: 259-302.

Driscoll, Paul, and Anya McGuirk. 1992. A class of separability flexible functional forms. Journal of Agricultural and Resource Economics 17: 266-276.

Edgerton, David L. 1993. On the estimation of separable demand models. Western Journal of Agricultural Economics 18: 141-146.

Edgerton, David L. 1997. Weak separability and the estimation of elasticities in multistage demand systems. American Journal of Agricultural Economics 79: 62-79.

Gao, X.M., Eric J. Wailes, and Gail L. Cramer. 1997. A microeconometric analysis of consumer taste determination and taste change for beef. American Journal of Agricultural Economics 79: 573-582.

Gorman, W.M. 1959. Separable utility and aggregation. Econometrica 27: 469-481.

Gorman, W.M. 1995. Two-Stage Budgeting. In Separability and Aggregation -- Collected works of W.M. Gorman, volume I, edited by C. Blackorby and A.F. Shorrocks. Oxford: Clarendon Press. 
Granger, Clive W.J., Maxwell L. King, and Halbert White. 1995. Comments on testing economic theories and the use of model selection criteria. Journal of Econometrics 67: 173-187.

Hanemann, Michael, and Edward Morey. 1992. Separability, partial demand systems, and consumer's surplus measures. Journal of Environmental Economics and Management 22: 241-258.

Hayes, Kathy J. 1988. Third-order translog utility functions. Journal of Business \& Economic Statistics 4: 339-346.

Jorgenson, Dale W., and Lawrence J. Lau. 1975. The structure of consumer preferences. Annals of Economic and Social Measurement 4: 49-101.

Jorgenson, Dale W., Daniel T. Slesnick, and Thomas M. Stoker. 1997. Two-stage budgeting and consumer demand for energy. In Welfare -- Volume 1: Aggregate Consumer Behavior, edited by D.W. Jorgenson. Cambridge, MA: The MIT Press.

Kinnucan, Henry W., Hui Xiao, Chung-Jen Hsia, and John D. Jackson. 1997. Effects of health information and generic advertising on U.S. meat demand. American Journal of Agricultural Economics 79: 13-23.

LaFrance, Jeffrey T. 1991. When is expenditure exogenous in separable demand models? Western Journal of Agricultural Economics 16: 49-62.

Lau, Lawrence J. 1978. Testing and imposing monotonicity, convexity, and quasi-convexity constraints. In Production Economics: A Dual Approach to Theory and Applications, volume 1, edited by M. Fuss and D. McFadden. Amsterdam: North-Holland.

Michalek, J. and M.A. Keyzer. 1992. Estimation of a two-stage LES-AIDS consumer demand system for eight EC countries. European Review of Agricultural Economics 19: 137-163.

Moschini, GianCarlo. 1992. A non-nested test of separability for flexible functional forms. Review of Economics and Statistics 74: 365-369.

Moschini, GianCarlo. 1998. The semiflexible almost ideal demand system. European Economic Review 42: 349-364.

Moschini, GianCarlo. 1999. Imposing local curvature conditions in flexible demand systems. Journal of Business \& Economic Statistics 17: 487-490.

Moschini, GianCarlo, and Daniele Moro. 1994. Autocorrelation specification in singular equation systems. Economics Letters 46: 303-309.

Moschini, GianCarlo, Daniele Moro, and Richard D. Green. 1994. Maintaining and testing separability in demand systems. American Journal of Agricultural Economics 76: 61-73. 
Newman, Peter. 1969. Some properties of concave functions. Journal of Economic Theory 1: 291-314.

Pollak, Robert A.. 1971. Conditional demand functions and the implications of separable utility. Southern Economic Journal 37: 423-433.

Schwarz, G. 1978. Estimating the dimension of a model. The Annals of Statistics 6: 461-464.

Spencer, Peter. 1997. Monetary integration and currency substitution in the EMS: The case for a european monetary aggregate. European Economic Review 41: 1403-1419.

Terrell, Dek. 1996. Incorporating monotonicity and concavity conditions in flexible functional forms. Journal of Applied Econometrics 11: 179-194.

Uzawa, Hirofumi. 1962. Production functions with constant elasticity of substitution. Review of Economic Studies 29: 291-299.

Yen, Steven T., and Terry L. Roe. 1989. Estimation of a two-level demand system with limited dependent variables. American Journal of Agricultural Economics 71: 85-98. 